\title{
Color Imagery of Skin Tone and Eyeglass Frames
}

\author{
Kuen-Meau Chen, Ying-Sin Lin, and Hsueh-Cheng Chou \\ Department of Industrial Design, National United University, \\ 1 Lien Da, Kung-Ching Li, Maioli 36003, Taiwan \\ $1 \mathrm{kmchen} 0721$ @gmail.com
}

\begin{abstract}
This study aimed to explore the colour imagery of skin tone and eyeglass frames, and whether this affects the preferences of consumers. Glasses are a focal point on the face and we wished to understand whether the frame colour along with skin tone affects the entire facial image. Many businesses provide suggestions on choosing frames that complement one's skin tone, but what is the basis of these recommendations? We conducted a questionnaire survey and analysed the results using two-way ANOVA and regression analysis. Results were as follows: (1) skin tone significantly influences preference; (2) the colour of eyeglass frames significantly influences preference; (3) the interaction of skin tone and frame colour does not significantly affect preference; (4) consumers with lighter skin tone tend to prefer black frames; (5) elegant, simple, fresh imagery significantly and positively influences preference. Our results showed that when consumers are choosing glasses, the colour relationship between skin tone and glasses frames does not significantly influence their decisions; however, when it comes to choosing the frames, consumers place more emphasis on their skin tone or colour preferences.
\end{abstract}

Keywords: Glasses design, colour imagery.

\section{Introduction}

\subsection{Motivation and Objectives}

In addition to correcting one's vision, eyeglasses have become an accessory capable of expressing one's individual style. Occasionally, the difference between skin tones and eyeglass frames is quite striking, such as when a black person wears white frames or a white person wears dark frames. Salespeople and websites dealing with eyeglasses often recommend that their customers select the correct color for their eyeglass frames as a marketing ploy, while consumers tend to maintain personal preferences. This paper explores the relationship between skin tone and the color of eyeglass frames with the following objectives:

1. To explore the emotional imagery evoked by the arbitrary combination of various skin tones and frames of different colors;

2. To understand the inclinations of consumers with regard to skin tones and the color of eyeglass frames. 
This research may provide a useful reference for eyeglass designers in the selection of colors.

\subsection{Scope and Limitations}

Hairstyle, facial characteristics, and the style of eyeglasses all play a role in the selection of eyeglasses. This study selected "skin tone" as the sole variable affecting one's decision in the selection eyeglass frames in the hope of eliminating factors that might otherwise confound the research results.

\section{$2 \quad$ Literature Review}

Stylish product design has become an important global trend. Style can be a unique characteristic, which is presented as an outward expression of oneself (Schmitt \& Simonson, 1997). Trends in product design have been steered from functionalism toward product semantics Krippendorff, 1996). Krippendorff and Butter (1984) defined product semantics as 1. "research that focuses on the meanings and symbols of objects in the psychological and social dimensions" and 2. "research on the forms and symbolic features of manmade objects in the users' environment and an application of such knowledge on industrial design" (Krippendorff \& Butter , 1991). Krampen (1996) claimed that every commodity (structures or products) has its own stylistic or connotative significance. A wide range of eyeglasses are available on the market and in addition to the function of holding optical lenses, the eyeglass frame also serves as an adornment that helps to express oneself and alter one's image. Eyeglass frames also indicate one's social status, professional appearance, and fashion acumen. Mature markets include a wide range of consumers with different preferences and needs. This study is based on the assumption that style can be analyzed using a recognition-coding system based on the emotional connotations shared by most people.

Designers provide strong and emotional design skills and uses provide their "experience skills". These two skills are the two factors which connects the design objects and the users. (Norman, 2004). According to Khaslavsky and Shedroff (1999), designers use enticement, relationship, and fulfillment to project sentimental expression. Designs are determined by the color, shape, and materials used in the product as well as the latest trends. From computer accessories and headphones to household appliances and vehicles, people consider the color of a product when making decisions related to purchasing behavior (Nagamachi, 1995).

Chen (2008) claimed that humans undergo physical and psychological changes when they see things and receive stimulation from the outside world. Skin tone and the color of the eyeglass frames are two important factors to be considered. When working on the design of spectacles, the matching of skin tones and the color of eyeglass frames can make a pronounced difference with regard to visual effect. This paper sought to identify the inclinations of the general public with regard to skin tones and the color of eyeglass frames. 


\section{$3 \quad$ Research Design}

This study progressed through the following three phases: First, we assembled sampling pre-tests by making a glossary of skin tones, the colors of eyeglass frames, and the terminology used to describe style. This led to the drafting of a questionnaire for a public opinion survey. Two-way ANOVA was used to analyze the inclinations and preferences of consumers with regard to the matching of skin tones and the color of eyeglass frames. In the third phase, we employed regression analysis to investigate the impressions that could influence the decisions involved in the selection of eyeglass frames with the aim of identifying the crucial elements believed to embody good style.

\subsection{Sampling of Skin Tones}

Samples were obtained from the skin-tone chart proposed by Felix von Luschan, an Austrian anthropologist, who classified human skin colors into 36 degrees, ranging from nearly pure white to black. The chart was then compared with the skin color distribution map by Italian geographer Renato Biasutti. The resulting map indicated that the skin tones of Taiwanese people range from 12-14. We used Photoshop to adjust the skin tones and color intensity in the sample images to the near-average complexion found in Taiwan, resulting in 15 skin samples. The questionnaire was administered to 14 college students with a major in design. The questionnaire included two questions: (1) What skin tone do you think can best represent the Taiwanese people? (2) Please choose three samples of "light, average, and dark" skin tones that are most typical of Taiwanese people. We then collected the questionnaires and selected Sample 5 (for light complexion), Sample 9 (for average complexion), and Sample 13 (for darker complexion) as representative samples (see Figure1).

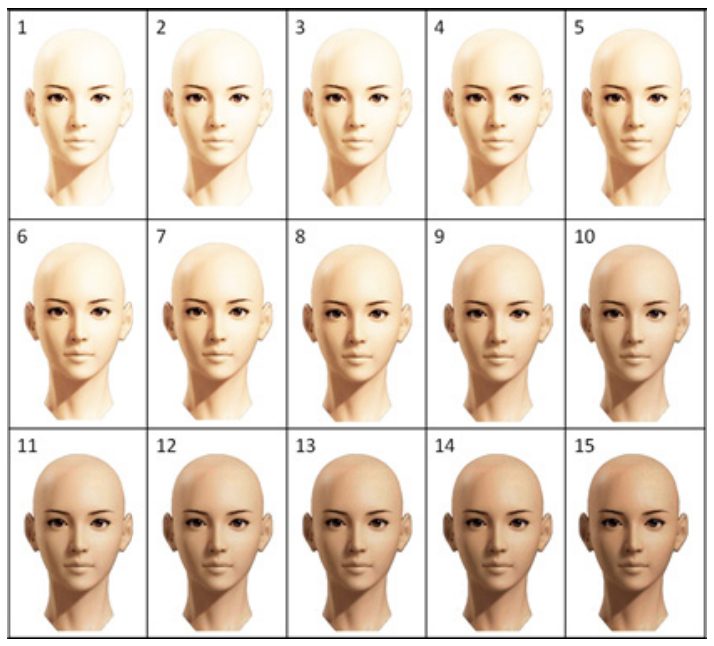

Fig. 1. Sampling of skin tones 


\subsection{Selection of Adjectives Related to Imagery}

This study assembled several comparative adjectives from a study on color recognition and the color of plastic eyeglass frames (Liang et al. 2010) and organized a conference of experts to discuss the issue. Feedback from the experts was used for the selection of the following eight pairs of adjectives appropriate for the glossary: (1) cool vs. warm; (2) gloomy vs. refreshing; (3) immature vs. mature; (4) dusky vs. bright; (5) lowly vs. noble; (6) serious vs. sprightly; (7) nerdy vs. glamorous; and (8) delightful vs. unlikeable.

\subsection{Colors Selection for Eyeglass Frames}

For the selection of the colors of eyeglass frames, we adopted the Practical Color Coordinate System (P.C.C.S) and selected a color every 60 degrees, with additional neutral tones (grey, black, and white). We made the samplings for eyeglass frames in accordance with the P.C.C.S-RGB-CMYK, and obtained a total of nine colors: Y, G, $\mathrm{gB}, \mathrm{V}, \mathrm{RP}, \mathrm{rO}$, grey, black, and white.

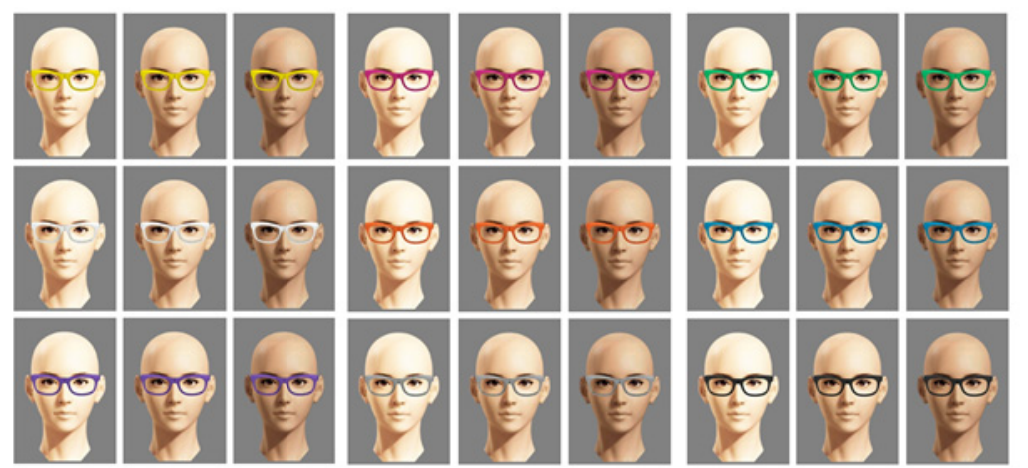

Fig. 2. Sampling of eyeglass-wearing icons

\subsection{Questionnaire Design and Testing}

Photoshop was used to apply the nine colors of eyeglass frames onto the three types of skin tone. The location where we put the eyeglass on the face was based on the standard adopted by most people who wear eyeglasses. This resulted in 27 samples $(9 * 3)$ (see Figure 2). This study employed a questionnaire survey to collect information from students. The order of testing ran as follows: (1) Assemble adjectives to describe skin tones and the color of eyeglass frames by recruiting ten college students majoring in design; (2) Assemble a sample of skin tones by recruiting 14 college students with a major in design; (3) Conduct a survey with 30 students to identify "emotional imagery" produced by matching skin tones and the color of eyeglass frames as well as "levels of preferences".

We then listed a sample icon on each page (27 icons in total), with eight scoring items. The color of the eyeglass frames was matched with the three skin tones (light, average, dark). This study employed a Osgood Sematic Differential, scoring ranged 
from 1 through 7. SPSS 20.0 was used for two-way ANOVA in exploring the degree of preferences and impressions perceived by consumers in the matching of skin tones and the color of eyeglass frames.

\section{$4 \quad$ Results and Analysis}

\subsection{Analysis of Two-Way ANOVA and Preference Levels}

We discerned that (1) the significance of "skin tone" ( $\mathrm{P}$ value $=0.000$ ) suggest that skin tone has a noticeable impact on one's preferences; (2) the significance of the "color of the eyeglass frame" ( $\mathrm{P}$ value $=0.000)$ shows that the color of the eyeglass frame has a noticeable impact on one's preferences; (3) the significance of the matching of "skin tone and color of eyeglass frames" ( $\mathrm{P}$ value $=0.903$, which is far above 0.05) does not reach significance, suggesting that "the matching of skin tone and the color of the eyeglass frames" does not have noticeable effects on one's preferences.

From the multiple comparisons of skin tone preferences, we discovered a noticeable difference between dark skin and the other two skin tones. Put differently, consumers were less attracted to the icons with darker skin, whereas the difference between light skin and average skin tone remained small.

Light skin was slightly preferred over average skin tones and black frames were preferred, followed by orange, blue, and white. The preferences for red, yellow, and green are clearly lower than the aforementioned colors for all three skin tones.

It was apparent that participants preferred light skin, followed by average and dark skins. With the exceptions of orange, red, and green colors, the participants show a higher preference for any color when matched with light skin. In addition, the preference for black frames was far higher than for any of the other colors.

\subsection{Regression Analysis}

This study conducted regression analysis on the seven adjective imageries and levels of preferences, producing three models to explain the phenomenon. Model 3 presents the highest explanatory power, which produced three adjective pairs, as follows: (1) gloomy vs. refreshing; (2) lowly vs. noble; (3) nerdy vs. glamorous. The predicted variable equation runs as follows:

$y=0.999+0.866 *($ noble $)+(-0.379) *($ glamorous $)+0.262 *($ refreshing $)$, illustrating the impact of the three adjective pairs on the participants in evoking an emotional response. "Noble" and "refreshing" exert positive effects on consumers whereas "glamorous" has a negative effect on perceptions of eyeglass frames.

\section{Conclusions}

It was discovered that skin tones and the color of eyeglass frames play a pivotal part in one's preferences, while the relationship between skin tone and the color of eyeglass frames has almost no influence. Darker skin had a less positive impression on the viewers, while fair skin was admired. The difference between light and average skin tones is smaller, because the preference level depended on the fairness of the skin. 
The participants showed a higher preference for all frames exhibited on light skin, regardless of the color. This confirms that skin tone, rather than the color of the eyeglass frame, had a greater impact on the viewers. The most popular color for eyeglass frames was black. The adjectives indicating preference were noble, quiet, and refreshing. These results provide a useful reference for further research and designers in the eyeglass industry for the selection of colors used in future design.

\section{References}

1. Schmitt, B., Simonson, A.: Marketing Aesthetics: The Strategic Management of Brands, Identity, and Image. Simon \& Schuster (1997)

2. Khaslavsky, J., Shedroff, N.: Understanding the seductive experience. Communications of the ACM 42(5), 45-49 (1999)

3. Krampen, M.: Semiotics in Architecture and Industrial/Product Design. In: Margolin, V., Buchanan, R. (eds.) The Idea of Design, 2nd Printing, pp. 89-103. The MIT Press, Massachusetts (1996)

4. Krippendorff, K.: On the Essential Contexts of Artifacts or on the proposition that Design is Making Sense (of Things). In: Margolin, V., Buchanan, R. (eds.) The Idea of Design, vol. 184, pp. 56-184. MIT Press, Massachusetts (1996)

5. Krippendorff, K., Butter, R.: Product Semantics: Exploring the Symbolic Qualities of Form. Innovation, The Journal of IDSA 3(2), 4-9 (1984)

6. Liang, C.C., Chen, K., Ho, C.H.: A Study on Color Emotion for Plastic Eyewear. In: New World Situation: New Directions in Concurrent Engineering, pp. 147-162 (2010)

7. Mitsuo, N.: Kansei Engineering: A new ergonomic consumer-oriented technology for product development. International Journal of Industrial Ergonomics 15, 3-11 (1995)

8. Norman, D.A.: Emotional design: Why we love (or hate) everyday things. Basic Books, New York (2004)

9. Chen, K.M., Wang, M.J.: The Influence of Single Colour Preference and Area Ratio on Dichromatic Colour Preference. Colour: Design \& Creativity (2), 5, 1-9 (2008) 